\title{
Primary recovery of lipase derived from Burkholderia sp. ST8 with aqueous micellar two-phase system.
}

\begin{abstract}
The partitioning and recovery of lipase derived from Burkholderia sp. ST8 strain was explored using temperature-induced aqueous micellar two-phase system (AMTPS) composed of single nonionic surfactant. Nonionic surfactant Triton X-114 and Pluronic series (triblock copolymer) were evaluated in terms of their clouding phenomenon (cloud-point temperature) and the performance of the lipase partitioning in these AMTPSs. Pluronic L81 showed the most optimum partition efficiency for the recovery of lipase to the micellar phase of the AMTPS. Based on the AMTPS which consisted of $24 \%(\mathrm{w} / \mathrm{w})$ Pluronic L81 and $0.5 \%(\mathrm{w} / \mathrm{w})$ potassium chloride $(\mathrm{KCl})$, the selectivity of lipase partitioned to bottom phase has been enhanced to 0.035 and the lipase was purified 7.2 fold. Furthermore, the lipase from the micellar phase was consecutively extracted to a new aqueous solution, with an aim of removing the surfactant from the purified lipase. It was attained by replacing the aqueous top phase from the primary recovery of AMTPS with a new potassium thiocyanate (KSCN) solution. The lipase was then recovered in the newly formed bottom aqueous phase which culminated in the yield of $89 \%$ and partition coefficients of 0.34 and 4.50 for lipase and surfactant, respectively. AMTPS offers a convenient and efficient method for the primary recovery of lipase with low cost, large loading capacity and the potential of linear scale up.
\end{abstract}

Keyword: Aqueous micellar two-phase system (AMTPS); Lipase; Recovery; Triblock copolymer; Pluronic; Partition. 